\title{
Notas taxonómicas sobre el género Avellinia Parl. (Poaceae: Poeae: Aveninae)
}

\section{Carlos Romero-Zarco' \& Llorenç Sáez ${ }^{2}$}

'Departamento de Biología Vegetal y Ecología, Universidad de Sevilla (Sevilla)

²Sistemàtica i Evolució de Plantes Vasculars (UAB) - Unitat Associada al CSIC. Unitat de Botànica, Dpt. Biologia Animal, Vegetal i Ecologia, Facultat de Biociències, Universitat Autònoma de Barcelona, 08193 Bellaterra, Barcelona

\section{Correspondencia \\ C. Romero Zarco \\ e-mail:zarco@us.es}

Recibido: 14 noviembre 2018

Aceptado: 20 noviembre 2018

Publicado on-line: noviembre 2018

\section{Taxonomic notes on the genus Avellinia Parl. (Poaceae: Poeae: Aveninae)}

Palabras clave: Taxonomía, Avellinia, Gramineae, Poaceae, Menorca.

Key words: Taxonomy, Avellinia, Gramineae, Poaceae, Menorca.
Durante la elaboración del manuscrito destinado al volumen XIX de Flora iberica (Castroviejo, 1986 en adelante) se ha estudiado la variabilidad morfológica de Avellinia festucoides (Link) Valdés \& H. Scholz en la Península Ibérica, Islas Baleares y países próximos de la cuenca mediterránea occidental. El género Avellinia Parl. se ha considerado hasta la fecha monoespecífico, pero su única especie conocida ha sido descrita por varios autores en diferentes géneros: Avena L., Bromus L., Festuca L., Koeleria L., Trisetaria Forssk., Trisetum, Pers. e incluso Vulpia C.C. Gmel., habiéndose acumulado una larga lista de sinónimos (23 se recogen en The Plant List). Recientemente se propuso su clasificación dentro el género Rostraria (Romero-Zarco, 1996), sin embargo las relaciones filogenéticas en el grupo formado por los géneros Koeleria, Rostraria Trin., Trisetaria y Trisetum, con algunas de cuyas especies está emparentado, son complejas (Quintanar \& al., 2007). En consecuencia, se impone por el momento el criterio de mantener un concepto morfológico tradicional para estos géneros, entre los cuales Avellinia está claramente delimitada por la especial configuración de sus lemas, que son arqueados y de dorso redondeado, y no aquillados como ocurre en el resto del grupo.

Avellinia festucoides se distribuye por casi toda la cuenca Mediterránea, a excepción del extremo SE (Valdés \& Scholz, 2009) y está introducida en Australia. El estudio del material de herbario ha revelado que la mayoría de las plantas del género estudiadas procedentes de la isla de Menorca destacan de las restantes poblaciones por presentar aristas de $3 \mathrm{~mm}$ o más (pueden alcanzar
4,3 mm) en las dos flores inferiores de la espiguilla, longitud aproximadamente igual o algo superior a la del lema correspondiente y claramente mayor que la observada en el resto del territorio de Flora iberica (hasta 2,2 mm), Figura 1. En diversas síntesis florísticas consultadas correspondientes a territorios no ibéricos, no se ha documentado la presencia de plantas con las aristas de más de 3 mm de longitud (Pignatti, 1982; Tison \& al., 2014). Las plantas menorquinas con aristas largas fueron descritas en el rango varietal por Font Quer (1919) y para ellas proponemos un tratamiento específico, que consideramos adecuado por corresponder a un ámbito geográfico concreto, junto con un claro carácter morfológico. Según R. Vogt (in litt.) no hay material original de la especie en el herbario del Jardíny Museo Botánico de Berlín (B). Aunque el tipo de la especie no ha sido localizado aún, asumimos que con toda probabilidad correspondería a la forma de aristas cortas, la única encontrada en el territorio portugués, de donde fue descrita la especie.

Entre el resto de las plantas estudiadas (herbarios: BC, BCN, FCO, G, HUAL, MAF, MGC, SALA, SANT, SEV y VAL, siglas según Index Herbariorum), solo escasas muestras del SE de Franciay deIN de África presentan aristas de tamaño algo mayor que el de las plantas peninsulares (2-3 $\mathrm{mm}$, rango documentado por Pignatti, 1982 y Tison \& al., 2014), pero siempre claramente más cortas que el lema correspondiente.

En relación con las plantas marroquíes distribuidas por Sennen, tipificamos aquí también dos de los nombres que este prolífico botánico usó para Avellinia festucoides: Avellinia warionis 


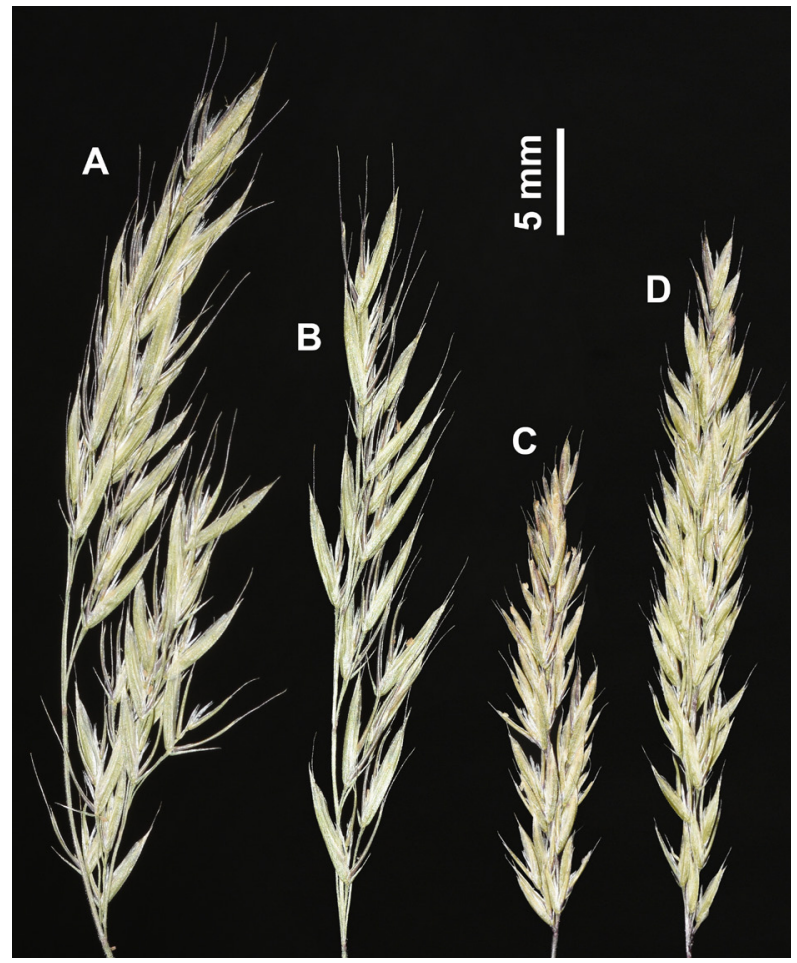

Figura 1. Inflorescencias de Avellinia longiaristata (A, B) y de $A$. festucoides (C, D).

Figure 1. Inflorescenses of Avellinia longiaristata $(A, B)$ and $A$. festucoides $(C, D)$.

A, B: Menorca, Ferreries, so n'Ermità, leg. P. C: Mallorca, Escorca, Coll des Prat, leg. L. Sát $<$, Barcelona, Torrelles de Llobregat, leg. L. Sáez.

Sennen \& Mauricio y Trisetum viciosorum Sennen \& Mauricio. Finalmente, en esta aportación esclarecemos la sinonimia de una planta baleárica, Trisetum balearicum Gand., cuya identidad ha estado envuelta de notable controversia y que, de acuerdo con nuestras investigaciones pertenece también a la típica Avellinia festucoides.

\section{Avellinia longiaristata (Font Quer) Romero Zarco} \& L. Sáez, comb. \& stat. nov.

Basiónimo: Avellinia michelii var. longiaristata Font Quer in Bol. Soc. Esp. Hist. Nat. 19: 273 (1919)

Indicación locotípica: "Menorca, en la cúspide del Monte Anclusa, de Ferrerías, en terreno arenoso"

Lectotipo: Menorca, S'Anclusa, terrenos arenosos, 14-V-1913, Font Quer (BC-68569), designado aquí.

Otros materiales estudiados. ESPAÑA. Islas Baleares, Menorca. S'Anclusa [31SEE82], Font-Quer (MA 9597); Ciutadella, 31SEE7728,
30-IV-1951, P. Montserrat (BCN 84206); íbidem, carretera [Maó-Ciutadella] km 40,3 [31SEE72], 30IV-1951, P. Montserrat (BCN 84205); Ferreries, so n’Ermità [31SEE82], 26-IV-2018, P. Fraga (L. Sáez, herb. pers.).

Por el momento se trata de un taxón endémico de Menorca, donde habita en terrenos arenosos, desde cerca del nivel del mar hasta $275 \mathrm{~m}$ de altitud, en pastizales xerofíticos y claros de formaciones arbustivas.

Avellinia warionis Sennen \& Mauricio in Sennen, Diagn. Nouv. 248 (1936)

Nombre aceptado: Avellinia festucoides (Link) Valdés \& H. Scholz in Willdenowia 36: 662 (2006)

Avellinia michelii var. warionis (Sennen \& Mauricio) Maire in Jahand. \& Maire, Cat. PI. Maroc 4: 935 (1941)

Indicación locotípica: "Maroc: Beni-Bu-Yahi, djebel Kerker, à Puerta Abada, vers 850 m."

Lectotipo: Maroc: Djebel Kerker, à Puerta Abada (Beni-Bu-Yahi), 22-IV-1934, frère Sennen \& hermano Mauricio (BC-Sennen 830947), designado aquí.

Isolectotipos: Maroc: Djebel Kerker, à Puerta Abada, 850 m (Bni-Bu-Yahi), 22-IV-1934, Sennen \& Mauricio, Sennen, PI. Espagne 1934, nº 9688 (BC 80941; G 309531; MAF 26267), designados aquí.

El ejemplar designado como lectotipo lleva, además de la etiqueta impresa del exsiccatum, otra manuscrita con los mismos datos de recolección. Se estudió en febrero de 1996 pero hasta la fecha no había sido publicada su tipificación.

Trisetum viciosorum Sennen \& Mauricio in Sennen, Diagn. Nouv. 244 (1936)

Nombre aceptado: Avellinia festucoides (Link) Valdés \& H. Scholz

Indicación locotípica: "Maroc: Atlas Rifain, meseta de Isaguen, $1600 \mathrm{~m}$."

Lectotipo: Maroc: Atlas Rifain, meseta de Isaguen, 1600 m, 20-IV-1934, Sennen \& Mauricio, Sennen, PI. Espagne 1934 nº 9612 (BC 80866), designado aquí.

Este nombre fue asignado a la sinonimia de Avellinia festucoides cuando se estudió el material de Marruecos (Romero-Zarco, 1996), pero hasta la fecha no había sido formalmente tipificado.

Trisetum balearicum Gand. in Bull. Soc. Bot. France 47: 141 (1900)

Nombre aceptado: Avellinia festucoides (Link) Valdés \& H. Scholz

Indicación locotípica: „Hab. in collibus et pinetis siccis maritimis, tam ad Sabina quam ad 
San Francisco" [Formentera]

Lectotipo: Baleares, Formentera, V-1899, M. Gandoger sn. (W-1899-0008821), designado aquí.

Este nombre no aparece en la revisión de Jonsell (1980) y según algunos autores (Gil \& Llorens, 2001), podría corresponder a Trisetaria panicea (Lam.) Pers., mientras que Bolòs \& Vigo (2001) indican explícitamente que desconocen la identidad de Trisetum balearicum Gand. Por su parte, en la base de datos en línea The Plant List (2013) se considera T. balearicum un sinónimo de Rostraria pumila (Desf.) Tzvelev.

El material del pliego W-1899-0008821, además de la etiqueta original antes indicada, incluye una etiqueta de revisión de Bengt Jonsell, quien en 1976 excluye que dicho material corresponda al género Trisetum e indica "Vulpia sp.". Posteriormente, C.A. Stace estudió este material de T. balearicum y consideró (etiquetas con fecha 21-VIII-1981) que corresponde a "Avellinia michelii (Savi) Parl." y que dicho material podría ser typus de la especie descrita por M. Gandoger. El material del pliego W-1899-0008821 ciertamente corresponde a una forma típica de Avellinia festucoides (aristas de 1-1,5 mm de longitud) por lo que Trisetum balearicum Gand. se debe incluir en su sinonimia.

\section{Agradecimentos}

Agradecemos a P. Fraga el muestreo de material del género Avellinia de Menorca y a los conservadores de los herbarios citados el préstamo del material consultado.

\section{Referencias}

Bolòs, O. \& J. Vigo (2001). Flora dels Països Catalans.
Vol. 4: 268-273. Ed. Barcino. Barcelona.

Castroviejo, S. (coord., 1986). Flora iberica. Plantas vasculares de la Península Ibérica e Islas Baleares. Real Jardín Botánico, CSIC, Madrid.

Font Quer, P. (1919). Adiciones a la flora de Menorca. Boletín de la Real Sociedad Española de Historia Natural 19: 268-273.

Gil, L. \& L. Llorens (2001). Plantes vasculars de I'llla de Formentera. ORCA. Catàlegs Florístics locals 8. Institut d'Estudis Catalans. Barcelona.

Index Herbariorum (1997 en adelante). New York Botanical Garden, Steere Herbarium. New York. http://sweetgum.nybg.org/science/ih/ [consultada en noviembre de 2018]

Jonsell, B. (1980). Trisetum L. En Tutin, T.G., V.H. Heywood, N.A. Burges, D.M. Moore, D.H. Valentine, S.M. Walters \& D.A. Webb. Flora Europaea 5 : 220224. Cambridge University Press, Cambridge.

Maire, R. (1953). Avellinia Parl. En R. Maire \& M. Weiller. Flore de l'Afrique du Nord 2: 341-343. Paris.

Pignatti, S. (1982). Flora d'Italia. Ed. Edagricole. Vol 3: 516. Bologna.

Romero-Zarco, C. (1996). Contribución al conocimiento de las gramíneas del $\mathrm{N}$ de Marruecos. Lagascalia 18(2): 310-321.

Tison, J.M., P. Jauzein \& H. Michaud (2014). Flore de la France méditerranéenne continentale. Conservatoire Botanique National Méditerranéen de Porquerolles. Naturalia Publications. Turriers.

The Plant List. Version 1.1 (2013). The Royal Botanic Gardens Kew \& Missouri Botanical Garden. http:// www.theplantlist.org/ [consultada en noviembre de 2018]

Valdés, B. \& H. Scholz; with contributions from RaabStraube, E. von \& Parolly, G. (2009). Poaceae (pro parte majore). Euro+Med Plantbase - the information resource for Euro-Mediterranean plant diversity. http://ww2.bgbm.org/EuroPlusMed/ [consultada en noviembre de 2018] 